\title{
A Review of Fuzzy Logic Model for Analysis of Computer Network Quality of Experience
}

\author{
Walter .B. Kihuya \\ Institute of Computer Science \\ and Information Technology, \\ Jomo Kenyatta University of \\ Agriculture and Technology, \\ Mombasa, Kenya.
}

\author{
Dr. Calvins Otieno \\ Institute of Computer Science \\ and Information Technology, \\ Jomo Kenyatta University of \\ Agriculture and Technology, \\ Nairobi, Kenya.
}

\author{
Dr. Richard Rimiru \\ Institute of Computer Science \\ and Information Technology, \\ Jomo Kenyatta University of \\ Agriculture and Technology, \\ Nairobi, Kenya.
}

\begin{abstract}
The estimation of the QoE provides valuable input in order to measure the user satisfaction of a particular service/application. Network QoE estimation is challenging as it tries to measure a subjective metric where the user experience depends on a number of factors that cannot easily be measured. All the Network analysis models can be divided into two major groups: qualitative and quantitative. In recent years many quantitative models have been developed in terms of quantitative measures i.e. use of scale of numbers between 1 to 5 to represent user perception of QoS. The challenge with this model is where user perception is subjective and not precise thus cannot be clearly measured using quantitative methods. On the other side qualitative models are in early stages of exploration. Little has been done on qualitative methods. Basing on previous studies, few models exists that measure qualitative analysis of computer network quality of experience. However none incorporated all the four parameters of integrity of service; throughput, delay, packet loss and jitter as parameters of network QoE. In this work, a literature survey is done on qualitative performance by use of a variety of variables, input and output linguistic terms. After a broad survey of the literature, we tend to propose a fuzzy logic model for analysis of computer network QoE. Likewise, the model combines all the four parameters of network integrity of service parameters since they are the primary factor for QoS quantification of any network [1]. Moreover, by using the fuzzy logic concept, the output linguistic terms shows the user perception about a product or a service (QoE) to certain levels by use of membership functions, in this case triangular membership function which shows the mapping of each linguistic term to certain range of values rather being precise to a particular value. By such means, the developed fuzzy logic model tends to accommodate some degree of uncertainty and vague network values to be used for analysis purposes. The concern is to advance the analysis and evaluation of quality of experience in computer networks by use of fuzzy logic concept. The target population for this model is the ISPs' clients. This will enable ISPs to have the best responsive measures to deal with clients' QOE parameters so as to meet the QOS as per SLAs. Keywords: fuzzy logic, ISPs (Internet Service Providers), quality of experience (QoE), Quality of service (QoS), SLAs (Service Level Agreement)
\end{abstract}

\section{INTRODUCTION}

As the service requirements of network applications shift from high throughput to high media quality, interactivity, and responsiveness, the definition of Quality of Experience (QoE) has become multidimensional.

QoE in the context of telecommunications networks is defined as the degree of delight or annoyance of the user of an application or service. It results from the fulfillment of his or her expectations with respect to the utility and / or enjoyment of the application or service in the light of the user's personality and current state [2]. QoE is defined by International Telecommunication Union (ITU) as the overall acceptability of an application or service, as perceived subjectively by the end-user. [3]
The information technology (IT) and electronics industries apply the QoE model to businesses and services since QoE depends on customer experience; assessments are compiled from large user group polls. QoE models are in two broad categories: Qualitative and Quantitative models. Among the most commonly used quantitative model is mean opinion score (MOS). The MOS is expressed as a single rational number, typically in the range $1-5$, where 1 is lowest perceived quality and 5 is the highest perceived quality. Other MOS ranges are correspondingly possible, depending on the rating scale that has been used in the underlying test. This model is thus quantitative in nature while user perception is subjective and not precise thus cannot be clearly measured using quantitative methods as it tends not to accommodate uncertainty [3]

Fuzzy logic is a problem solving methodology that provides a simple way of definite conclusions from vague and 
imprecise information. Fuzzy set theory was first introduced by Zadeh in 1965 . He was motivated by observing that human reasoning can utilize concepts and knowledge that don't has well defined boundaries [4].

In recent years, the number and variety of applications of fuzzy logic have increased significantly. The applications range from consumer products such as cameras, camcorders, washing machines, and microwave ovens to industrial process control, medical instrumentation, decision-support systems, portfolio selection and network analysis.

In the case of Network analysis, all Network analysis models can be divided into two major groups: qualitative and quantitative. Qualitative metrics do not possess quantitative values and cannot be measured by numerical numbers. In that case, linguistic terms are used to evaluate performance of qualitative metrics [5]

QoE estimation is challenging as it tries to measure a subjective metric where the user experience depends on a number of factors that cannot easily be measured. In subjective assessment, quality is judged by users. Vagueness occurs when users have different interpretation of the same word (linguistic values) like poor, better.

\section{THEORETICAL REVIEW}

\section{Network Quality of experience (QoE)}

Quality of experience (QoE) is defined as the overall acceptability of an application or service, as perceived subjectively by the end-user [3]

The concept of QoE is used to measure user satisfaction level as shown in Figure 2.1. QoE includes complete endto-end system ranging from users, terminal, customer premises network \& core network and access network to service infrastructure.

Therefore based on the definition of QoE, network QoE can be defined as the overall acceptability of the network service(s) as perceived subjectively by the end-user.

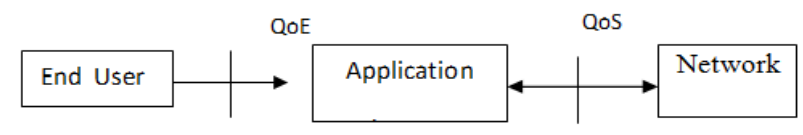

Figure2. 1: Relationship between QoS and QoE

\section{Fuzzy logic}

Fuzzy logic is a natural, continuous logic patterned after the approximate reasoning of human beings [6]. As a theory mathematical discipline, fuzzy logic reacts to constantly changing variables [6].It challenges traditional logic by not being restricted to the conventional binary computer values of zero and one. Instead, fuzzy logic allows for partial truths and multivalued truths [6].Fuzzy logic is especially advantageous for problems that cannot be easily represented by mathematical modeling because data is either unavailable or incomplete or the process is too complex [6].The real world language used in fuzzy control enables engineers to incorporate ambiguous, approximate human logic into computers using linguistic modeling, as opposed to mathematical modeling, greatly simplifies the design and modification of a fuzzy logic system [6].

Fuzzy set theory [7] was developed to address contexts in which decision Makers need to accurately analyze and process information that is imprecise in nature.

Fuzzy sets provide a conceptual framework, as well as an analytical tool to solve real World problems where there is a lack of specific facts and precision. Human semantics are embedded in the meaning of fuzziness and comparison [8] On the other hand; the usage of multi granularity linguistic information can eliminate the difference from evaluators [9] An objective of fuzzy logic has been to make computers think like people [6]. Fuzzy logic can deal with the vagueness intrinsic to human thinking \& natural language and recognizes that its nature is different from randomness [6].Using fuzzy logic algorithms could enable machines to understand and respond to vague human concepts such as hot, cold, large, small, etc. It also could provide a relatively simple approach to reach definite conclusions from imprecise information [6].

\section{Integrity of service}

Integrity of service involves maintaining the consistency, accuracy, and trustworthiness of data over its entire life cycle. Data must not be changed in transit, and steps must be taken to ensure that data cannot be altered by unauthorized people for example, in a breach of confidentiality. These measures include file permissions and user access controls. Some means must be in place to detect any changes in data that might 
occur as a result of non-human-caused events such as an electromagnetic pulse (EMP) or server crash. Some data might include checksums, even cryptographic checksums, for verification of integrity. Backups or redundancies must be available to restore the affected data to its correct state [10].

\section{ISPs (Internet Service Provider)}

Internet Service Provider is a company that provides customers with Internet access. Data may be transmitted using several technologies, including dial-up, DSL, cable modem, wireless or dedicated high-speed interconnects. [3]

Among the largest national and regional ISPs are AT\&T WorldNet, IBM Global Network, MCI, Netcom, UUNet, and PSINet. Examples of ISPs in Kenya include Zuku, Safaricom, Airtel, Orange, Faiba internet, Access Kenya, Internet Solution etc.

\section{Quality of Service (QoS)}

Quality of Service (QoS) is the degree of conformance of the service delivered to a user by a provider in accordance with an agreement between them [3]

In order to assess QoS of any network efficiently, network and service related performance metrics should be identified carefully. The available literatures in this context analyze the diverse characteristics of such metrics. Each service has some crucial parameters that greatly influence its performance. This has been studied in detail in [11]

These studies suggest that for QoS evaluation of any network, it is useful to choose metrics, which are interrelated to QoE parameters. As a result, it is possible to assess the network QoE by simply applying the values of the network QoS parameters.

\section{Traditional Approaches to analysis of network QoE}

Mean Opinion Score (MOS) is a numerical value which is used as an index for expressing the QoE. The standard scores for MOS are available in ITU-T Recommendation P.800 [3]. These ranking grades, which are shown in Table below, were originally applied for the quality assessment of telephone transmission. Today, these judgment scales are used to evaluate the QoE for different applications and services. For instance, MOS was used for evaluation of users' experience of web browsing [12]

Table2. 1 : Opinion scores recommended by ITU-T.

\begin{tabular}{|l|l|}
\hline Score & Sequence quality \\
\hline 5 & Excellent \\
\hline 4 & Good \\
\hline 3 & Fair \\
\hline 2 & Poor \\
\hline 1 & Bad \\
\hline
\end{tabular}

Today, compared to the objective assessment of QoS parameters, the subjective assessment of QoE has attracted an increasing attention. Traditional mechanism commonly used in assessments is a survey in which users' opinions are asked on a five-point scale and later on average the results collected to calculate the Mean Opinion Score (MOS) value to be used for analysis/evaluation. Each user rates the perceived quality based on different quality scales ranged from 1 to 5 , where 5 refers to the excellent perceived quality and 1 indicates the lowest experienced quality. The results from MOS will be an indicator for service providers to understand the user's perception and define proper thresholds for each quality scale.

This approach still comes with its share of challenges. For example, the MOS scores 1, 2, 3, 4, and 5 correspond to Bad, Poor, Fair, Good, and Excellent experiences, respectively. It is not surprising that people may have different interpretations of a similar linguistic term for instance "Bad" and give different ratings even though they have the same experience in a test. Likewise, each linguistic term is mapped to a particular value thus not accommodating imprecision.

These observations prompted scholars to further investigate on the causal relationship between smart technology and QoE measurement. Fuzzy logic technique is one of the smart technologies in use. Others include neural network, Support Vector Machine, Naive Bayes, Decision Tree etc. Several researches have been done on fuzzy logic in relation to QoE though little has been done on fuzzy logic model for analysis of computer networks quality of experience. 


\section{III.METHODOLOGICAL REVIEW}

\section{Fuzzy logic models for analysis of Qualitative performance:}

Several researches have been done on fuzzy logic in relation to quality performance though little has been done on fuzzy logic model for analysis of quality of experience.

The study in [13] proposed a Fuzzy logic aggregation of wireless sensor network data for smart traffic light control. This approach uses smart traffic control systems (STCS) to make traffic routing decisions. STCS use real time data and mimic human reasoning thus prove promising in vehicle traffic control. This presents a smart traffic light controller using fuzzy logic and wireless sensor network (WSN). The approach is designed for an isolated four way roundabout. It employed fuzzy logic to control the lights and determine how the green light will be assigned for each approach. The WSN collected the traffic data in real time. This data is aggregated and fed into a fuzzy logic controller (FLC) in form of two inputs - traffic quantity (TQ) and waiting time (WT) for each approach. Based on the inputs, the FLC then computes an output priority degree (PD) that controls green light assignment. Using the $\mathrm{PD}$, an algorithm is formulated that assigns green light to the lane with highest PD. The cycle continues until all approaches get green.

In [14] a research study on a Fuzzy Logic System for Evaluating Quality of Experience of Haptic-based Applications was proposed. The proposed taxonomy was modeled with a fuzzy logic system and finally was tested by a Mamdani fuzzy inference system. In the mentioned study, by making some assumption like rule selection and membership function selection, the effect of different perception measures parameters such as rendering quality, physiological and psychological was studied. Here, fuzzy logic system was applied for objective measuring of QoE parameters.

The research work in [15] exhibited QoE estimation for web service selection using a Fuzzy-Rough hybrid expert system. A methodology to estimate the quality of web services based on a fuzzy-rough hybrid algorithm is proposed. The estimated web QoE is used to select the most performing service among different web services. Fuzzy expert systems are good at making decision with imprecise information; however, they cannot automatically formulate rules that they require for making the decisions. Therefore, a fuzzy-rough hybrid expert system is proposed in this study where rough set theory is used to define the rules necessary for the fuzzy expert system. Three QoS parameters: reliability, execution time (in seconds), and availability (in seconds) were measured during the performance of the tests. Input linguistic terms were: Low, Medium and High. The output linguistic terms in use were: Bad, Poor, Fair, Good and Excellent.

The research work in [16] proposed analysis of Quality of Experience by applying Fuzzy logic: A study on response time. In this work, with a fuzzy perspective, the effect of response time variation in a network on the quality perceived by users is shown. Later, shows how by applying fuzzy techniques the linguistic terms and the users' perception can be translated into quantitative values. The main objective of this project was to analyze the fuzziness of QoE in order to provide more understandable user perception. This included proposing response time performance criteria that correlate well with QoE measurement result presented by fuzzy concepts. The proposed methodology provides a fuzzy relationship between QoE and Quality of Service (QoS) parameters. To identify this fuzzy relationship a new term called Fuzzi ed Opinion Score (FOS) representing a fuzzy quality scale is introduced. A fuzzy data mining method is applied to construct the required number of fuzzy sets. Then, the appropriate membership functions describing fuzzy sets are modeled and compared with each other. The proposed methodology intended to assist service providers for better decision-making and resource management [16].

In [17], an efficient algorithm for transmitting packet for better quality of service in adhoc mobile network was proposed. In this study, Fuzzy Self Organizing Map (FSOM) provide very efficient algorithmic tools for transmitting packet in an efficient manner by taking the most efficient route, the bandwidth, latency and range network parameters are considered to determine how good is the data delivered. The results indicated that fuzzy logic can guarantee QoS of every packet in the network. Incorporation of fuzziness in the input and output of the proposed model was seen to result in better performance. Input variables were only three properties: low, normal, and high. The output variables were poor, good and excellent.

In [18] a fuzzy logic based approach is in use for maintaining VoIP Quality in a network which is affected by many network factors (packet loss, packet delay, and jitter).In this case, Resource Reservation Protocol application was configured to 
control Token Bucket Algorithm and the simulation experiments were carried out with Opnet. In addition, comparison between Token Bucket with and without Quality of Service aimed at measuring network factors was performed. In this paper, building Fuzzy Token Bucket System consisted of three variables (Bandwidth Rate, Buffer Size, and New Token) in order to improve Token Bucket Shaper output variable (New Token) by Fuzzy Stability model for Voice over IP quality maintaining. The linguistic values in use for each variable were: Buffer Size \{VL, L, M, H, and VH\}, Bandwidth Rate \{VL, L, AL, BA, AV, AA, BH, H, and $\mathrm{VH}\}$ and New Token $\{\mathrm{VL}, \mathrm{L}, \mathrm{BA}, \mathrm{AV}, \mathrm{AA}, \mathrm{H}$, and $\mathrm{VH}$ \}

The study in [19] revealed the analysis of the impact of different network QoS parameters on users perceived video QoE for VoD (Video-on-Demand) services. Network parameters in use included: Packet loss rate, Burst packet loss and Jitter. The input linguistic terms involved were Very annoying, slightly annoying, Imperceptible, Annoying and perceptible but not annoying. The output linguistic terms in use were Very annoying, slightly annoying, Imperceptible, Annoying and perceptible but not annoying. This study proposed a methodology based on a fuzzy expert system to objectively estimate the video QoE. To validate the methodology, the developed system was integrated as part of a monitoring tool in an industrial IPTV (Internet Protocol Television) test bed and compared its output with standard Video Quality Monitoring (VQM). The evaluation results show that the proposed video quality estimation method based on fuzzy expert system can effectively measure the network impact on the QoE.

In [19], a methodology and a system based on fuzzy expert system to estimate the impact of network conditions (QoS) on the QoE of video traffic were presented. At first, subjective tests to correlate network QoS metrics with participants' perceived QoE of video traffic was conducted. Secondly, a No Reference method based on fuzzy expert system to estimate the network impact on the video QoE was proposed. The membership functions of the proposed fuzzy system were derived from normalized probability distributions correlating the QoS metrics with QoE. A simple methodology to build the fuzzy inference rules was proposed. The system was evaluated in two different sets of experiments. The estimated video quality showed high correlation with the subjective QoE obtained from the participants in a controlled test. The evaluation results show that the proposed video quality estimation method based on fuzzy expert system can effectively measure the network impact on the QoE. [19] In this study, six video clips of different types (sports, movie, animation, and interview) were used and generated 228 sample video clips which were constructed with different network level perturbations.

These video clips were constructed by streaming from a server to client and correspondingly introducing perturbation through emulated network. Three QoS parameters for perturbation were selected; packet loss, jitter and packet loss burstiness, which were considered promising for the mapping of QoS to QoE for video traffic. [19].

The variation of these QoS metrics impacts the quality of the delivered video and, consequently, the user satisfaction level. The objective was to design and implement a method to estimate the variation of the user satisfaction level in function of the network QoS conditions [19].

In the subjective test, different video clips were presented to the 25 participants who rated each video clip according to the perceived impairment giving one of the following scores:

_ Imperceptible (score 5): Excellent conditions.

_ Perceptible but not annoying (score 4): Good conditions.

_ Slightly annoying (score 3): Fair conditions.

_ Annoying, and (score 2): Poor conditions.

_ Very annoying (score 1): Bad conditions.

From the subjective test, a learning set that consisted of the mapping between the participants' scores and the QoS metrics for each of the considered video clips was built. A probabilistic approach to correlate QoS metrics to the participants' scores was used. Therefore, for every QoS metric, five different probability distribution functions (pdf) was built, one function per QoE score that provide the variation of the participants' ratio (\%) with the QoS metric for a specific QoE score. This probabilistic information was changed into a fuzzy set by dividing the pdf by its peak value i.e. normalized pdf [20].

The fuzzy set, which has the same form as that of the original pdf, was converted into an equivalent triangular or trapezoidal fuzzy set by using a curve fitting method as demonstrated in [21].The triangular or trapezoidal fuzzy set represents the membership functions for the different QoS metrics as illustrated below. 


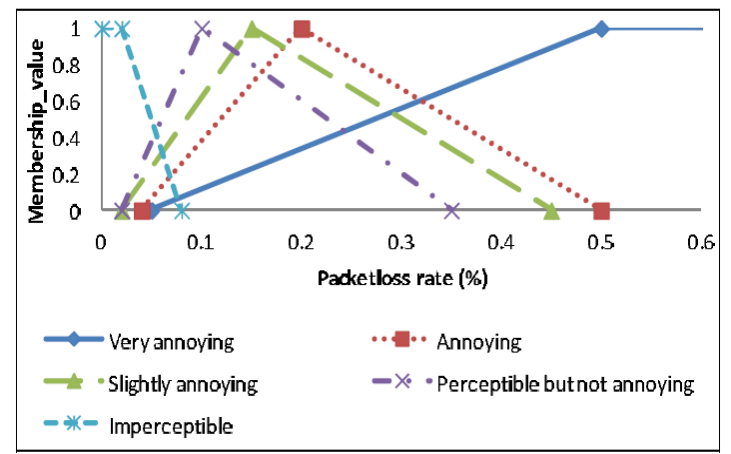

Figure2. 2: Membership functions for packet loss rate metric. [19]

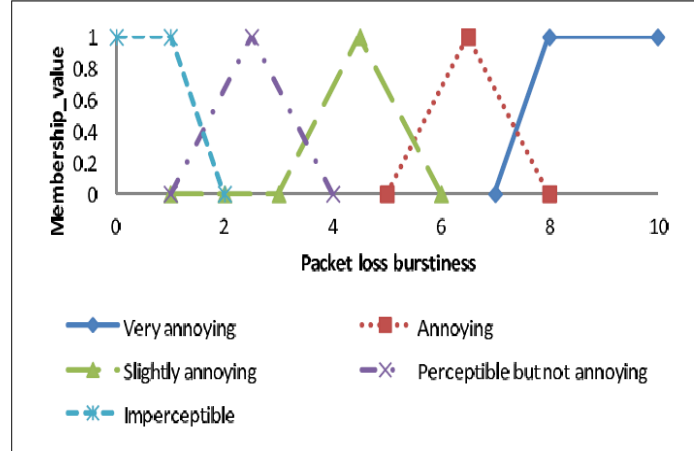

Figure2. 3: Membership functions for packet loss burstiness metric. [19]

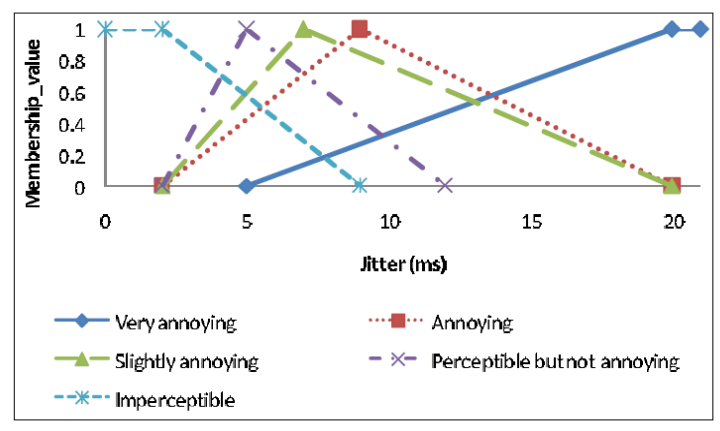

Figure2. 4: Membership functions for jitter metric. [19]

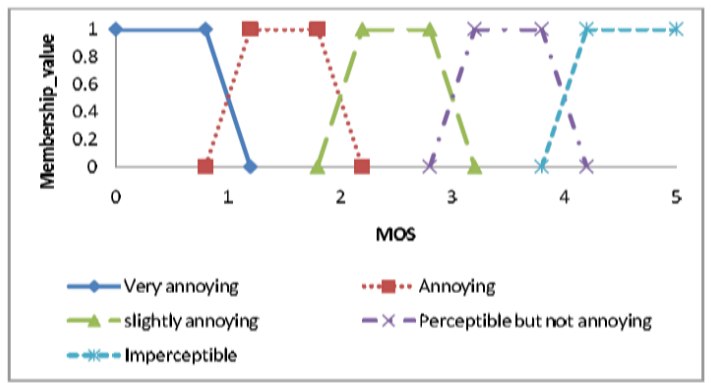

Figure2. 5: Memberships function for the estimated MOS: eMOS. [19]

\section{Critiques of the existing Literature relevant}

\section{to the study:}

Based on the literature of the work cited, it's a clear indication that each research work used different types of parameters and linguistic terms.

For QoS evaluation of any network, it is useful to choose metrics, which are interrelated to QoE parameters. As a result, it is possible to assess the network QoE by simply applying the values of the network QoS parameters. The QoE parameters include Accessibility, Retain_ability and Integrity of Service each of them having its corresponding QoS parameters under consideration [10].

Few of the research work considered the underlying QoSrelated parameters, which are linked to the integrity of service QoE parameters but none of them considered all the four parameters (Throughput, delay, jitter and packet loss).

\section{Research Gap}

Few models exist that analyze qualitative analysis of network QoE though most of them have limited network parameters such as [19] used packet loss, packet loss burstiness /delay and jitter metrics. None of the models incorporated all the four Network QoE metrics for Integrity of Service (throughput, delay, packet loss and jitter) as major parameters of network QoE. Therefore, this review is inspired to address this gap by presenting an alternative approach of analyzing underlying QoS related parameters under integrity of service QoE parameters incorporating all the four Network QoE metrics for Integrity of Service i.e. throughput, delay, jitter and packet loss by use of fuzzy logic concept. These four parameters are considered to be the primary factors which affect any computer networks [10]. The concern is to advance the analysis and evaluation of quality of experience in computer networks by use of fuzzy logic concept. 


\section{Fuzzy Logic Model for Analysis of computer Networks Quality of Experience.}

Based on the literature work covered on fuzzy logic technique for analysis of qualitative performance by use of various variables, input and output linguistic terms, this survey tends to propose a fuzzy logic model for analysis of computer networks QoE.

Five input linguistic terms are identified: Very High, High, Medium, Low and Very Low. This approach is in reference to [11], whereby exhausting the concept of fuzzy membership function, the working ranges of network parameters are set using the term set \{Very Low (VL), Low (L), Medium (M), High (H), and Very High $(\mathrm{VH})\}$ rather than simply stating an acceptable range. This enables the integration of network and/or service uncertainty and dynamics together.

Likewise, centering on [22] indicates that the permissiveness of fuzziness in the human thought process suggests that much of the logic behind thought processing is not traditional two valued logic or even multivalued logic, but logic with fuzzy truths. Middle values for all the terms can be introduced and the concept of linguistic hedges can be used to identify for each fuzzy linguistic variable such as very low, very high and so on.

Moreover, in relation to [23], its clearly illustrated that linguistic hedge (linguistic modifier) is a function that alters the membership function of the fuzzy set associated to the linguistic label, obtaining a definition with a higher or lower precision depending on the case. Two of the most well-known modifiers are the concentration linguistic hedge "very" and the dilation linguistic hedge "more-or-less." Grounding on these facts, it prompted for the use of the stated input linguistic terms.

Five output linguistic terms are defined to describe the opinion scores: Excellent, Good, Fair, Poor and Bad. These values are based on International Telecommunication Union (ITU-T) recommendation for evaluation of QoS.

Four QoS parameters are used: delay, jitter, packet loss and throughput.

QoS parameters are factors that can affect the quality of service. These parameters are very important in Service Level Agreement (SLA) monitoring. Moreover, recent research shows the effect of variation of QoS parameters on the level of user satisfaction. Packet loss, delay (latency) and jitter are some of the most important parameters [24].

Therefore, determining a unified network QoS value can simplify the process of network QoE evaluation. The mappings between these QoS and QoE parameters are presented below:

Table5. 1: Mapping between QoE and QoS Related parameters [25]

\begin{tabular}{|l|ll|}
\hline \multirow{2}{*}{ QoE parameters } & \multicolumn{2}{|c|}{$\begin{array}{c}\text { Underlying QoS-related } \\
\text { parameters }\end{array}$} \\
\hline \multirow{4}{*}{ Accesibility } & $\bullet$ & Unavailability \\
& $\bullet$ & Security \\
& $\bullet$ & Activation \\
& $\bullet$ & Access \\
& $\bullet$ & Coverage \\
& $\bullet$ & Slocking \\
Retainability & & \\
\hline \multirow{3}{*}{ Integrity of Service } & $\bullet$ & Detime \\
& Connection loss \\
& $\bullet$ & Delay variation/Jitter \\
& $\bullet$ & Packet loss \\
\hline
\end{tabular}

\section{Conceptual Framework}

The QoE parameter under considerations is Integrity of Service having four underlying QoS-related parameters: Throughput, Delay, Jitter and packet loss. These are the primary factors for QoS quantification of any network as indicated by [1]

Table2. 2: Conceptual Framework derived from [10]

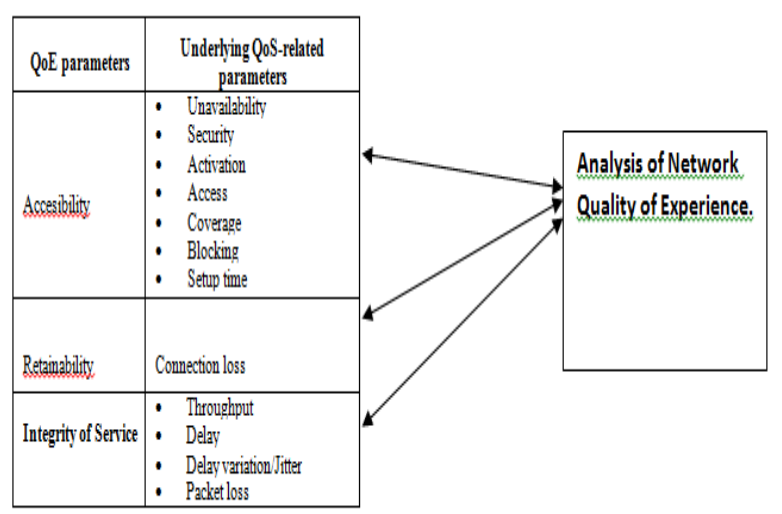


The above mappings between Network Integrity of Service QoE and QoS related parameters when manipulated in matlab environment effects in to below Conceptual Framework model:

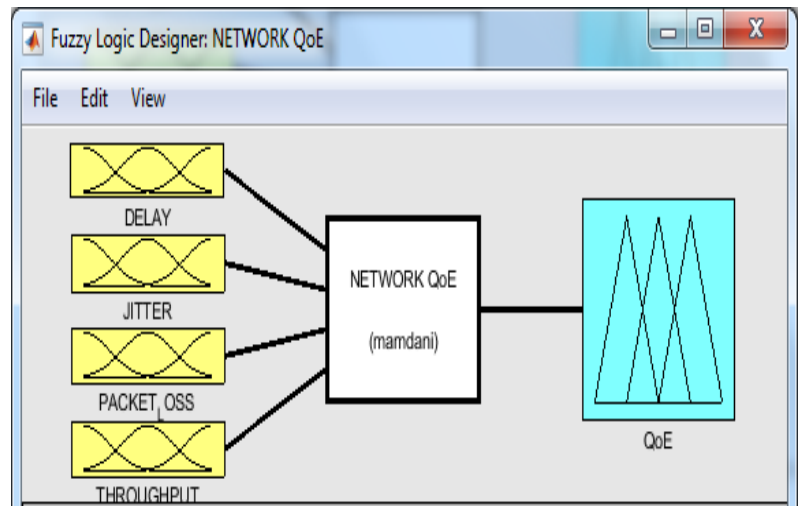

Figure2. 6: Conceptual Framework model for mappings between Network Integrity of Service QoE and QoS related parameters.

The model presents the following four attributes under network Integrity of service QoE:

Delay: Refers to an average time needed for a packet to reach from source to destination [19]. This parameter is intrinsic to communications, since the end points are distant and the information will consume some time to reach the other side. Delay is also referred as to latency. Delay time can be increased if the packet face long queues in the network (congestion), or crosses a less direct route to avoid congestion.

Jitter: Jitter is the variation in the packet inter-arrival delay [19]

It involves the delay variation and is introduced by the variable transmission of delay of the packets over the network. This can occur because of routers' internal queues behavior in certain circumstances for instance; flow congestion, routing changes, etc. This parameter can seriously affect the quality of streaming audio and/or video.

To handle jitter, it is needed to collect packets and hold them long enough until the slowest packets arrive in time, rearranging them to be played in the correct sequence.

Packet Loss: happens when one or more packets of data being transported across the internet or a computer network fail to reach their destination [19]. Wireless and IP networks cannot provide a guarantee that packets will be delivered at all, and will fail to deliver (drop) some packets if they arrive when their buffers are already full. This loss of packets can be caused by other factors like signal degradation, high loads on network links, packets that are corrupted being discarded or defect in network elements. Some transport protocols such as Transfer Control Protocol (TCP) make delivery control by receiving acknowledgements of packet receipt from the receiver. If packets are lost during transfer, TCP will automatically resend the segments which were not acknowledged at the cost of decreasing the overall throughput of the connection.

Throughput: refers to the number of bits received during a time unit [19]

Throughput is the amount of data which a network or entity sends or receives data, or the amount of data processed in one determined time space. It's basic unit of measures is bits per second (bit/s or bps). The throughput can be lower than the input strain due to losses and delays in the system. Throughput is a good measure of the channel capacity of a communications link.

\section{IV.METHODOLOGY:}

Fuzzy logic methodology in use involves the following steps:

a. Defining the linguistic variables and terms (Initialization).

b. Constructing the membership functions (Initialization).

c. Constructing the rule base (Initialization).

d. Converting the crisp input data to fuzzy values using the membership functions (Fuzzification).

e. Evaluating the rules in the rule base (Inference)

f. Combining the results of each rule (Inference).

g. Converting the output data to non-fuzzy values (Defuzzification).

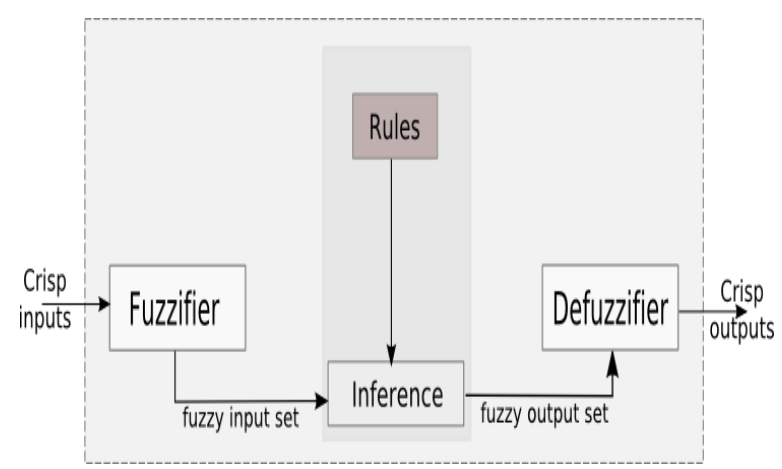

Figure3. 1: Fuzzy Logic System 


\section{Initialization}

This process involves Defining the linguistic variables and terms.

Constructing the membership functions (MF) is done at this stage. The process involves determining a curve that defines how each point in the input space is mapped to a membership value (or degree of membership) between 0 and 1. The input space is sometimes referred to as the universe of discourse. A triangular membership function is used in this work to obtain the degree of membership for each linguistic term. According to [26], Triangular membership functions are simple and therefore facilitate easy computation. As they are also piecewise linear they provide a linear mapping of the universe of discourse in the case of a look-up table method. For instance:

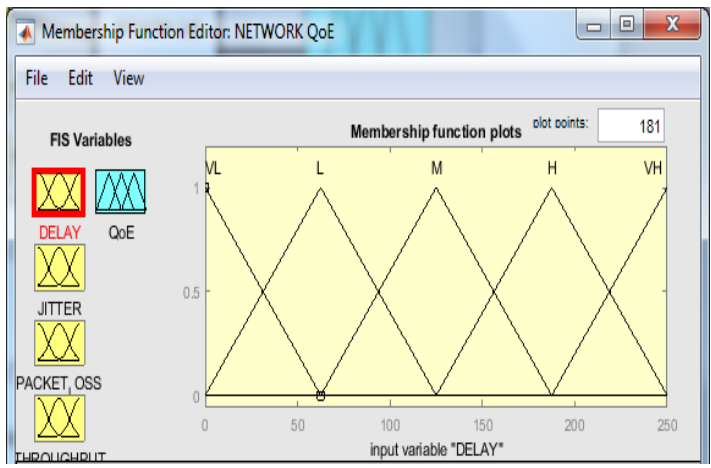

Figure4. 1: Developed membership function plots for Delay input linguistic term.

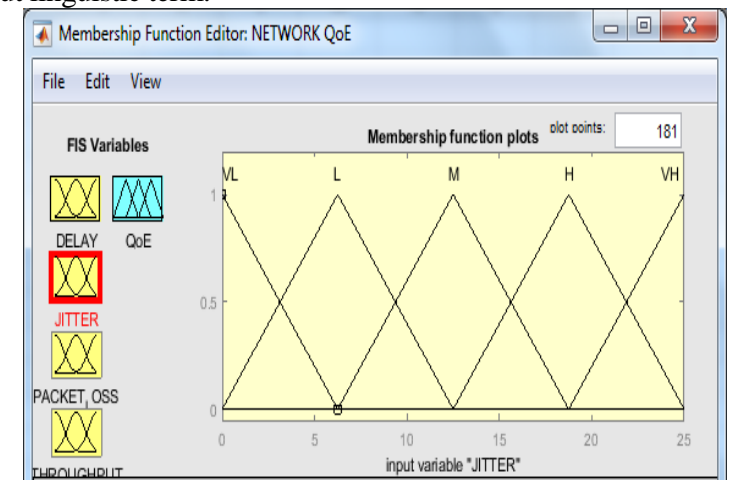

Figure4. 2: Developed membership function plots for Jitter input linguistic term.

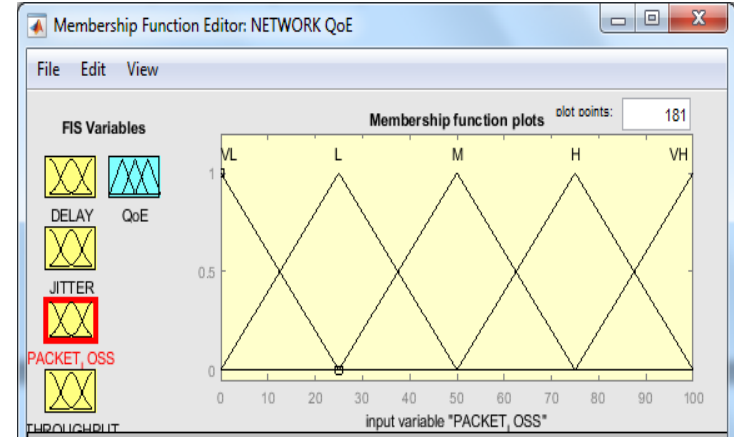

Figure4. 3: Developed membership function plots for Packetloss input linguistic term.

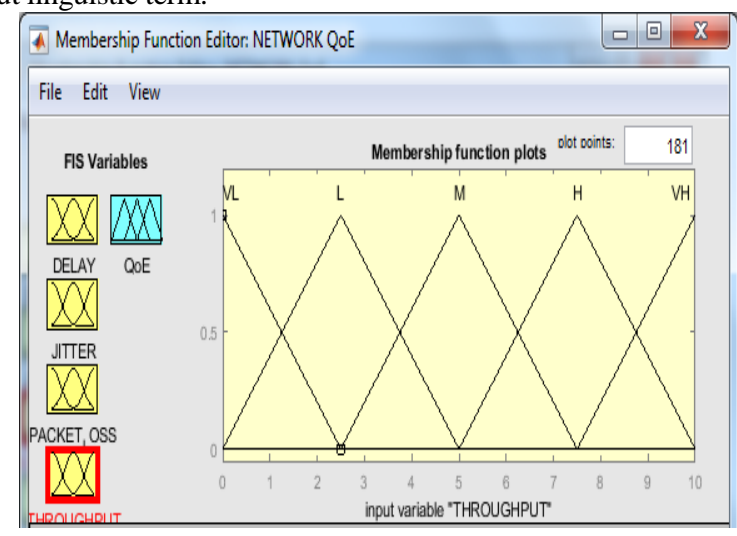

Figure4. 4: Developed membership function plots for Throughput input linguistic term.

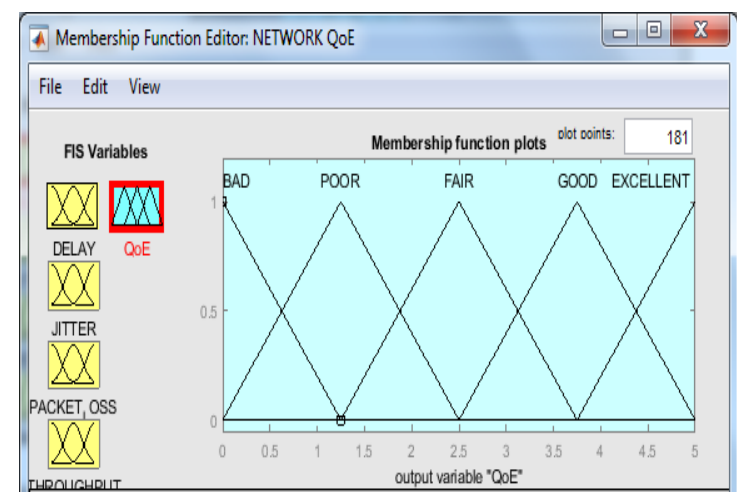

Figure4. 5: Developed membership function plots for different output linguistic terms.

Moreover, the initialization phase involves constructing the rule base. The identified Five linguistic terms for use i.e. "Excellent", "Good", "Fair", "Poor", and "bad" inclusive of the Four variables for network integrity of service QoE parameters i.e. Throughput, Delay, Delay variation/Jitter and packet loss results into 625 rules $\left(5^{\wedge} 4\right)$.

The rules were further dropped to 240 rules basing on expert knowledge by discarding the illogical rules thus remaining with logical rules to make rational decisions.

The illogical is as a result whereby some conditions cannot 
exist at the same time for instance in rule 1 of the 625 rules indicates:

1. If delay is very low, jitter is very low, packet loss is very low and throughput is very low then User Satisfaction N/A.

This rule is N/A thus illogical since when delay, jitter and packet loss are very low then throughput is supposed to be high or very high in ideal network situation as these three variables which are supposed to make the throughput very low, their existence too are very low not to certain levels to affect the network throughput to match being very low.

\section{Fuzzification:}

This phase involves converting the crisp input data to fuzzy values using the membership functions. This is achieved by Fuzzifier component of the fuzzy Logic System.

Firstly, a crisp set (subset elements of the set, definitely do belong to the set), of input data are gathered and converted to a fuzzy set (sets whose elements have degrees of membership) by using fuzzy linguistic variables, fuzzy linguistic terms and membership functions. This step is known as fuzzification [6].

\section{Inference:}

This stage involves evaluating the rules in the rule base. Each rule follows the order to fulfill certain condition. The logical 240 rules are interpreted one after the other. This is achieved by Fuzzy Inference system component of the Fuzzy Logic System. In this work, Mamdani fuzzy inference system is used to achieve the inferencing in the developed framework. The Fuzzy set operator "AND" is used to aggregate the output of each rule.

The results of each rule are combined at this phase. The matched fuzzy rules are then used in the defuzzification process.

The logical operator "AND" is selected for connecting the inputs in this experiment since the operator returns logical 0 (false) if even a single condition in the expression is false in an ideal situation [27].For instance in the rule below:
Example 1:

If delay is very low, jitter is very low, packet loss is very low and throughput is very high then User Satisfaction EXCELLENT:

In an ideal situation, when delay, jitter and packet loss are very low then throughput is very high as the network suffers no hitches thus resulting to user satisfaction being excellent.

\section{Defuzzification of the Output:}

The linguistic variables and terms are matched, fuzzy rules generated and output results obtained for each parameter are aggregated into one crispvalue through defuzzification.

This process involves producing a quantifiable result in Crisp logic, given fuzzy sets and corresponding membership degrees.

Moreover this process maps a fuzzy set to a crisp set. It is typically needed in fuzzy control systems. These will have a number of rules that transform a number of variables into a fuzzy result, that is, the result is described in terms of membership in fuzzy sets [28]

After the inference step, the overall result is a fuzzy value. This result should be defuzzified to obtain a final crisp output. This is the purpose of the defuzzifier component of a FLS.

Weighted average method technique is applied in this work because of its computational efficiency. It's formed by weighting each function in the output by its respective maximum membership value.

\section{RECOMMENDATION.}

Based on the review, it is greatly recommended to adopt Frameworks that have capabilities to accept vague and subjective values for analysis and decision making based on certain concepts or methodology for instance Fuzzy logic as user satisfaction is subjective in nature.

Moreover, in order to assess QoS of any network efficiently, network and service related performance metrics should be identified carefully.

In summary, there is a necessity to include all the four parameters which are linked to the integrity of service since 
they are considered to be the primary factors affecting any computer networks [10].

\section{BIBLIOGRAPHY}

[1] Y. Chen, T. Farley and N. Ye, "QoS Requirements of Network Applications on the Internet.," Information Knowledge • Systems Management Vol4, p. 55-76, 2004.

[2] K. Brunnstrom, S. A. Beker, K. D. Moor, A. Dooms, S. Egger and e. al., "Qualinet White Paper on Definitions of Quality of Experience," in Qualinet White Paper on Definitions of Quality of Experience Output from the fifth Qualinet meeting, Novi Sad,, 2013.

[3] ITU-T, "Methods for Subjective determination of transmission quality," p.800, 1996.

[4] M. Mehrdad and N. Abbas, "Supplier Performance Evaluation Based On Fuzzy Logic.," International Journal of Applied Science and Technology.1(5), pp. 257-265, 2011.

[5] U. Ezutah and Y. Kuan, "Supply Chain Performance Evaluation and challenges," American Journal of Engineering and Applied Sciences, pp. 202-211, 2010.

[6] L. Zadeh, "Fuzzy Sets," Information and Control Vol 8, pp. 338-353, 1965.

[7] S. Shruti and R. Mudholkar R, "Evaluation of teacher 's performance using Fuzzy Logic Techniques.," International Journal of Soft Computing and Engineering, 3(2), pp. 306-320, 2013.

[8] Zadeh, "A Computational approach to fuzzy quantifiers in natural language.," Computers and Mathematics with Applications 9(1), pp. 149-84, 1983.

[9] M. Mehrdad and N. A. Abbas, "Supplier Performance Evaluation Based On Fuzzy Logic.," International Journal of Applied Science and Technology.1(5), pp. 257 -265., 2011.

[10] F. Farid, S. Shahrestani and C. Ruan, "A Fuzzy Logic Approach for Quality of Service Quantification in Wireless and Mobile Networks.," in 10th IEEE International Workshop on Performance and Management of Wireless and Mobile Networks., Edmonton, Canada, 2014.

[11] F. Farnaz, S. Seyed and C. Ruan, "A Fuzzy Logic Approach for Quality of Service Quantification in Wireless and Mobile Networks.," in 10th IEEE International Workshop on Performance and Management of Wireless and Mobile Networks, Edmonton, Canada, 2014.

[12] J. Shaikh, M. Fiedler and D. Collange, " Quality of Experience from user and network perspectives.," https://doi.org/10.1007/s12243-009-0142-x, no. December 2009, p. 47-57, 2010.

[13] R. Hawi, G. Okeyo and M. Kimwele, "Techniques for Smart Traffic Control : An In-depth Review," Techniques for Smart Traffic Control : An In-depth Review. 4(7), p. 566573., 2015.

[14] A. Hamam, M. Eid, A. E. Saddik and N. D. Georganas, " A Fuzzy Logic System for Evaluating Quality of Experience of Haptic-Based Applications.," A Fuzzy Logic System for Evaluating Quality of Experience of Haptic-Based Applications. (October 2016). https://doi.org/10.1007/978-3540-69057-3, pp. 1-9, 2008.

[15] J. Pokhrel, ". Intelligent quality of experience (QoE) analysis of network served multimedia and webcontents.," Analyse intelligente de la qualité d ' expérience ( QoE ) dans les réseaux de diffusion de contenu Web et Multimédia., pp. 49-155, 2015.

[16] S. M. Ataeian and M. J. Darbandi, "Analysis of Quality of Experience by applying Fuzzy logic A study on response time," no. June, 2011.

[17] O. F. W. Onifade, "Better Quality of Service Management With Fuzzy Logic In Mobile Adhoc Network," vol. 6, no. 1, pp. 59-68, 2013.

[18] M. E. A. Ebrahim and H. A. Hefny, "Fuzzy Logic based Approach for VoIP Quality Maintaining," vol. 9, no. 1, pp. 537-542, 2018.

[19] P. Jeevan, "Intelligent quality of experience (QoE) analysis of network served multimedia and webcontents.," Analyse intelligente de la qualité d ' expérience ( QoE ) dans les réseaux de diffusion de contenu Web et Multimédia., pp. 49-155, 2015.

[20] B. S. De-lima and N. F. Ebecken, "A comparison of models for uncertainty analysis by the finite element method, Finite Elements in Analysis and Design, Vol 34,no.2," in Finite Elements in Analysis and Design, Las Vegas, USA, ScienceDirect, 2000, pp. 211-232.

[21] J. Pokhrel, F. Lalanne, A. Cavalli and W. Mallouli, "QoE estimation for web service selection using a FuzzyRough hybrid expert system".

[22] L. Zadeh, "Some reflections on the anniversary of Fuzzy sets and systems.," Journal of Fuzzy sets and systems, 100(2), pp. 1-3, 1999.

[23] A. S. Omar, M. Waweru and R. Rimiru, "Fuzzy Logic and Qualitative Performance Evaluation of Supply Chain Management," ISSN (e): 2319 - 1813 ISSN (p): 2319 1805, pp. 56-63, 2015. 
International Journal of Computer Applications Technology and Research

Volume 8-Issue 05, 201-212, 2019, ISSN:-2319-8656

[24] M. Fiedler, "A Generic Quantitative Relationship between Quality of Experience and Quality of Service," no. April, pp. 36-41, 2010.

[25] F. Farid, S. Shahrestani and C. Ruan, "A Fuzzy Logic Approach for Quality of Service Quantification in Wireless and Mobile Networks," pp. 629-636, 2014.

[26] E. Herbert, K. Thomas and M. Manfred, "A 12b General Purpose fuzzy logic controller chip," IEEE TRANSACTIONS ON FUZZY SYSTEMS, VOL 4, NO 4, pp. 460-475, 1996.
[27] mathworks, "www.mathworks.com/help/matlab/ref/logicaloperatorsshortci rcuit.html?searchHighlight=logical\%20operators\&s_tid=doc_ srchtitle," 193 2019. [Online]. Available:

https://www.mathworks.com/help/matlab/ref/logicaloperators shortcircuit.html?searchHighlight=logical\%20operators\&s_tid $=$ doc_srchtitle.

[28] W. V. Leekwijck and E. Kerre, "Fuzzy Sets and Systems," Defuzzification: criteria and classification., pp. 159 - 178, 1999. 